Louisiana State University

LSU Digital Commons

Faculty Publications

Department of Physics \& Astronomy

4-8-2009

\title{
Neutron production from beam-modifying devices in a modern double scattering proton therapy beam delivery system
}

\author{
Angélica Pérez-Andjar \\ University of Wisconsin School of Medicine and Public Health \\ Wayne D. Newhauser \\ University of Texas MD Anderson Cancer Center \\ Paul M. Deluca \\ University of Wisconsin School of Medicine and Public Health
}

Follow this and additional works at: https://digitalcommons.Isu.edu/physics_astronomy_pubs

\section{Recommended Citation}

Pérez-Andjar, A., Newhauser, W., \& Deluca, P. (2009). Neutron production from beam-modifying devices in a modern double scattering proton therapy beam delivery system. Physics in Medicine and Biology, 54 (4), 993-1008. https://doi.org/10.1088/0031-9155/54/4/012

This Article is brought to you for free and open access by the Department of Physics \& Astronomy at LSU Digital Commons. It has been accepted for inclusion in Faculty Publications by an authorized administrator of LSU Digital Commons. For more information, please contact ir@lsu.edu. 


\title{
Neutron production from beam-modifying devices in a modern double scattering proton therapy beam delivery system
}

\author{
Angélica Pérez-Andújar ${ }^{1,3}$, Wayne D Newhauser ${ }^{2}$, and Paul M DeLuca Jr ${ }^{1}$ \\ Angélica Pérez-Andújar: perezandujar@wisc.edu \\ ${ }^{1}$ University of Wisconsin, School of Medicine and Public Health, 750 Highland Avenue, 4111 \\ HSLC Madison, WI 53705-2221, USA \\ ${ }^{2}$ Department of Radiation Physics, Unit 94, The University of Texas MD Anderson Cancer Center, \\ 1515 Holcombe Blvd, Houston, TX 77030, USA
}

\begin{abstract}
In this work the neutron production in a passive beam delivery system was investigated. Secondary particles including neutrons are created as the proton beam interacts with beam shaping devices in the treatment head. Stray neutron exposure to the whole body may increase the risk that the patient develops a radiogenic cancer years or decades after radiotherapy. We simulated a passive proton beam delivery system with double scattering technology to determine the neutron production and energy distribution at $200 \mathrm{MeV}$ proton energy. Specifically, we studied the neutron absorbed dose per therapeutic absorbed dose, the neutron absorbed dose per source particle and the neutron energy spectrum at various locations around the nozzle. We also investigated the neutron production along the nozzle's central axis. The absorbed doses and neutron spectra were simulated with the MCNPX Monte Carlo code. The simulations revealed that the range modulation wheel (RMW) is the most intense neutron source of any of the beam spreading devices within the nozzle. This finding suggests that it may be helpful to refine the design of the RMW assembly, e.g., by adding local shielding, to suppress neutron-induced damage to components in the nozzle and to reduce the shielding thickness of the treatment vault. The simulations also revealed that the neutron dose to the patient is predominated by neutrons produced in the field defining collimator assembly, located just upstream of the patient.
\end{abstract}

\section{Introduction}

Protons and heavier charged particles offer the potentially significant advantage for radiation therapy of a dramatically improved physical dose distribution compared to indirectly ionizing particles such as photons or neutrons and lighter charged particles such as electrons (Wilson 1946). This results because most proton interactions occur by means of Coulombic interactions with atomic electrons (Gottschalk et al 1993). In particular, the large mass difference results in minimal deflection of the proton. The vast numbers of such interactions, each imparting very little energy loss, result in an almost continuous slowing down of the

(C) 2009 Institute of Physics and Engineering in Medicine

${ }^{3}$ Author to whom any correspondence should be addressed. 
protons. The clinical advantages of proton therapy are apparent (Suit 1992, Gragoudas et al 2002, Lodge et al 2007, Brada et al 2007).

However, not all proton interactions are due to electromagnetic interactions with atomic electrons. In fact, non-elastic interactions of the proton with atomic nuclei produce a broad spectrum of secondary neutrons as well as other spallation products. Non-elastic interactions occur with a probability of approximately 1-2\% per cm of material traversed (Janni 1982, ICRU 1993, ICRU 1998, ICRU 2007). Neutrons of high energy and considerable penetrability are generated in the treatment head and may reach the patient and shielding barriers of the treatment room. Neutron exposures are a predominant consideration in the shielding design of the treatment room and may pose long-term carcinogenic risk to patients. Thus, knowledge of the neutron emission rate from the nozzle is crucial to the shielding design of the treatment head and treatment vault.

Two system types, passive and active (dynamic), are commonly used to deliver a proton beam treatment (Chu et al 1993, Miller 1995, ICRU 1998). In passive systems, the proton range and fluence are varied by directing the beam through a spinning range modulation wheel (RMW). The RMW, together with a second scattering foil that spreads the beam laterally, produces a spread-out Bragg peak (SOBP). Generally, the materials employed for range modulation are of low atomic number $(Z)$ such as polymethyl methacrylate (PMMA), polycarbonate or aluminum (Koehler et al 1975, Chu et al 1993, Newhauser et al 2007). For lateral scattering, higher- $Z$ materials such as lead are used. Also a system of collimators shapes the proton field laterally and a range compensator (or bolus) can be employed to shape the distal surface of the field. Recently, several companies have developed treatment nozzles with double scattering systems (Matsuda et al 2006). In some such systems a RMW modulates the beam at the same time when it spreads it laterally (Gottschalk et al 1991). After the RMW there is a second scatterer that further spreads the beam laterally. This second scatterer is made of a set of inner and outer disks. The outer section is made of a low- $Z$ material while the inner is of high- $Z$ material. The outer low- $Z$ region compensates for the variable range shift caused by the inner high- $Z$ component. With this approach, the dose distribution is laterally uniform and the beam's penetration remains constant at off-axis locations.

Conversely in active systems (or spot scanning systems), a proton pencil beam with variable energy, and hence range, is manipulated such that there is no need for scattering foils, aperture collimation nor range compensators. It had been stated that scanned beams produce a tenth of the neutron exposure of passive beam delivery (Schneider et al 2002), although more recent studies (Tayama et al 2006, Fontenot et al 2008) suggest that the difference is smaller. In fact, the literature about neutron production in active systems is sparse. Passively scattered proton therapy systems, the focus of this work, are of particular interest because the majority of operating proton therapy centers rely exclusively on passively scattered beam delivery methods.

In passive systems, as presented previously, the proton beam interacts with energy degraders, scatterers and collimators. The bombarding beam loses significant energy in these components making them the major sources of secondary neutron production (Agosteo et al 
1998, Schneider et al 2002, Yan et al 2002, Polf and Newhauser 2005). These components are absent in some active beam delivery systems, thus suppressing neutron production. Schneider et al (2002) reported that the spot scanning system at the Paul Scherrer Institute (PSI) confers 1/10 the neutron dose $100 \mathrm{~mm}$ from the beam central axis than does the passive scattering system at the National Accelerator Center (NAC) in South Africa. The results from the NAC proton treatment facility appear atypical. The reported neutron equivalent dose values per therapeutic dose ${ }^{4}$ were significantly larger than those reported in more recent studies for other passive systems. These differences have not been resolved in the literature but may be traceable to the dose reporting methods as noted by Zheng (2007a). In addition, using Monte Carlo simulations, Agosteo et al (1998) estimated $10^{-3}$ Gy per therapeutic Gy for deep-seated tumors treatments due to neutrons and photons for active scanning systems. For passive systems the dose was of $10^{-2}$ Gy per therapeutic Gy, again this study was also based on the NAC passive system. Yan et al (2002) reported for the three passive treatment systems at the Harvard Cyclotron Laboratory a neutron equivalent dose of approximately $10^{-3} \mathrm{~Sv}$ per therapeutic dose at 0 degree with respect to the beam direction, $0.5 \mathrm{~m}$ from isocenter. The higher neutron equivalent doses were found for the largest angles closer to the treatment nozzle with a maximum of $1.5 \times 10^{-3} \mathrm{~Sv}$. Recently, neutron equivalent doses of the same magnitude as the ones reported by Agosteo et al (1998) for active systems were reported by Tayama et al (2006) for the passive system at the Proton Medical Research Center (PMRC) at Tsukuba.

Recently Zheng et al (2007a, 2007b, 2008) used Monte Carlo simulations to study stray neutrons from the double scattering passive beam delivery system at the M. D. Anderson Cancer Center. In one of their studies (Zheng et al 2007a), they estimated the neutron dose equivalent at various distances from isocenter. For forward angles, the neutron dose equivalent per therapeutic dose decreased with distance from isocenter. At back angles as the distance increases the dose increased. Presumably this was due to the neutron production from the passive beam spreading devices upstream of isocenter. Thus at back angles (larger than $90^{\circ}$ ) the detecting surfaces are in fact closer to the nozzle and therefore closer to the main source of neutrons.

The objective of this work was to explore the sources of secondary neutrons, the neutron energy fluence, its associated absorbed dose per therapeutic Gy and the neutron absorbed dose per source particle for a contemporary passively scattered proton therapy system. The beam spreading system modeled in this work closely resembles that of The University of Texas M. D. Anderson Cancer Center (Newhauser et al 2007, Zheng et al 2007a, 2007b). The neutron energy spectrum and absorbed dose were investigated for this system as a function of neutron emission. The neutron production was characterized separately for each of the major beam-modifying components inside the treatment unit. The study utilized the Monte Carlo simulation technique to predict the radiation fields.

\footnotetext{
${ }^{4}$ Therapeutic dose in Gy refers to the absorbed dose to the tumor in the central part of the SOBP.
} 


\section{Methods and materials}

\subsection{Neutron absorbed dose and energy spectra determination}

2.1.1. Monte Carlo simulations-The University of Texas M. D. Anderson Cancer Center proton beam delivery nozzle served as the basis for our model of a passive proton double scattering system. Specifically, it was used as a guide for the overall dimensions and the positions of the RMW, second scatterer and collimating system (Zheng et al 2007a, 2007b). However, we designed the RMW and second scatterer following the methods of Gottschalk et al (1991). This approach allowed for a design that was also representative of a second commercial proton therapy system (IBA, Belgium). Radiologically thin components, which produce minimal lateral scattering, energy loss and nuclear interactions, such as monitor chambers were not included because we believe they make minor contributions to neutron production in comparison to other components and also are specific to a given nozzle design.

To estimate the neutron production and subsequent transport, the Monte Carlo code package MCNPX developed by Los Alamos National Laboratory was used (Pelowiz 2005). This code allows simulations of the design of the desired geometry as well as the selection of the type of physics models that are appropriate for the type of particles to be simulated. While MCNPX can create and transport a large variety of particles, one can choose to transport only those of interest for a given situation. In the present case, only protons and neutrons were transported. In MCNPX, the sampled nuclear reaction cross-section data for most materials came from evaluated nuclear data libraries for energies from 0 to $150 \mathrm{MeV}$ (Pelowiz 2005, Chadwick et al 1999). At energies above $150 \mathrm{MeV}$, physics models were used to estimate the interaction cross-sections. In this work, the physics model, employed was the intranuclear cascade model originally described by Bertini (1963). The cross-section information was combined from evaluated data and nuclear models using the 'mix and match' option in MCNPX. Hence, for any given particle's transport, the code used the data from the evaluated tables, when available, up to their energy limit and then used the physics models. Also, for particle transport, the energy cutoff used for protons and neutrons in this study was $1 \times 10^{-3} \mathrm{MeV}$ and $0 \mathrm{MeV}$, respectively. The MCNPX code has been used extensively for research applications in proton therapy and benchmarked against measurements in numerous previous investigations (Heérault et al 2005, Koch and Newhauser 2005, Fontenot et al 2005, Newhauser et al 2005, 2007, Titt and Newhauser 2005, Tayama et al 2006, Polf et al 2007, Koch et al 2008, Titt et al 2008, Taddei et al 2008).

2.1.2. Geometry characteristics-A schematic diagram of the nozzle simulated is shown in figure 1 (Newhauser et al 2007, Zheng 2007a, 2007b). It consists of an RMW, a second scatterer, a set of collimators and a retractable snout with a final field-defining aperture. Table 1 presents the distances of the different nozzle components with respect to the RMW. The RMW was modeled as layers of lead and PMMA of various thicknesses, table 2, while the second scatterer was modeled as having geometry made of annuli of PMMA and disks of lead, table 3. The same scatterer was used for all simulations. The collimator system and snout materials were modeled as copper. The collimator system 
thicknesses are listed in table 1 . Notice that the geometry simulated did not include the nozzle enclosure components which would attenuate the neutron fields somewhat. The internal collimators were modeled such that the inner aperture had dimensions of $300 \times 300$ $\mathrm{mm}^{2}$ to avoid edge-scattering effects (Titt et al 2008) with the uncollimated therapeutic proton field of $180 \times 180 \mathrm{~mm}^{2}$, taken from the $50 \%$ to $50 \%$ profile width. Meanwhile the final collimator aperture was varied for the different sets of simulations, using $50 \times 50 \mathrm{~mm}^{2}$, $100 \times 100 \mathrm{~mm}^{2}$ and $150 \times 150 \mathrm{~mm}^{2}$ apertures. The thickness of the nozzle's collimating components were fixed for all field sizes, table 1 . To obtain SOBP widths of $50 \mathrm{~mm}, 100$ $\mathrm{mm}$ and $150 \mathrm{~mm}$ in depth, several simulations were combined, where each simulation included a single step of the RMW, which where modeled as right circular cylinders with variable thicknesses. The SOBP flatness in depth was achieved by varying the fluence weights of the individual RMW steps. Together, these adjustments provided field sizes with volumes of high dose regions of size $50 \times 50 \times 50 \mathrm{~mm}^{3}, 100 \times 100 \times 100 \mathrm{~mm}^{3}$ and $150 \times$ $150 \times 150 \mathrm{~mm}^{3}$.

In addition to the nozzle components, the simulated geometry included a water phantom whose front face was located at isocenter, $3.06 \mathrm{~m}$ from the RMW and $200 \mathrm{~mm}$ from the retractable snout. The water phantom had a radius of $150 \mathrm{~mm}$ and was $300 \mathrm{~mm}$ thick. Eight neutron-detecting surfaces were modeled at $4.06 \mathrm{~m}$ from the RMW and at angles of $0^{\circ}$ and $5^{\circ}$ as well as from $15^{\circ}$ to $90^{\circ}$ in $15^{\circ}$ increments. These angles are formed from the high- $Z$ to low- $Z$ interface in the RMW with respect to the primary proton beam direction. Each detecting surface was the front circular plane of a right circular cylinder $250 \mathrm{~mm}$ in diameter oriented facing the RMW. For all simulations, a $200 \mathrm{MeV}$ proton pencil beam was initiated upstream of the RMW on the central axis of the nozzle, interacting first with nozzle components, and then stopping in the water phantom. Some of these interactions produced neutrons, particularly in the RMW. Therefore, the neutron energy fluence was scored at each detecting surface and used to determine the neutron energy distribution and the neutron absorbed dose as a function of neutron emission angle.

2.1.3. Computational aspects-To obtain a set of data with good relative statistical uncertainty it was necessary to run simulations with as many as $150 \times 10^{6}$ source protons, which is similar to previous studies of relevance to this work (Zheng et al 2007a, 2007b, Fontenot et al 2005). The simulations were run on a cluster (GLOW 2004) that comprised 66 computer nodes with two $2.8 \mathrm{GHz}$ Intel Xeon CPUs per node and 29 nodes with two Intel Xeon CPUs of $3.20 \mathrm{GHz}$. Each simulation took approximately $270 \mathrm{cpu}$-hours.

2.1.4. Neutron absorbed dose and neutron energy spectra-The fluence of neutrons at each detecting surface was scored in energy bins ranging from $1.10 \times 10^{-5}$ to $200 \mathrm{MeV}$. The bin energy boundaries up to $150 \mathrm{MeV}$ were taken from the International Commission on Radiation Units and Measurements (ICRU) Report 63 (2000). Neutron absorbed dose was calculated on the assumption of secondary charged particle equilibrium and using the kerma approximation, or

$$
D_{\mathrm{n}}=K=\int_{0}^{\infty} \Phi_{\mathrm{n}}\left(E_{\mathrm{n}}\right) k\left(E_{\mathrm{n}}\right) \mathrm{d} E_{\mathrm{n}}
$$


where $D_{\mathrm{n}}$ is the absorbed dose (in Gy), $\Phi_{\mathrm{n}}\left(E_{\mathrm{n}}\right)$ is the neutron fluence (neutrons $\mathrm{cm}^{-2}$ proton $^{-1}$ ) for a given energy and $k\left(E_{\mathrm{n}}\right)$ is the kerma coefficient for the same energy (fGy $\mathrm{cm}^{2}$ ) at neutron energy $E_{\mathrm{n}}$. The assumption of secondary charge particle equilibrium is an approximation that is appropriate to the purpose of this study.

Kerma coefficients below $150 \mathrm{MeV}$ neutron energy were taken from ICRU Report 63. The kerma coefficients above $150 \mathrm{MeV}$ were estimated by fitting the ICRU data from 100 to 150 $\mathrm{MeV}$ with a third degree logarithmic extrapolation. This process created 10 bins that were equally spaced in the logarithm of neutron energy.

Additionally, the proton dose at the center of the SOBP, the so-called therapeutic dose in units of Gy, was obtained for each SOBP and field size studied. To accomplish this, the same nozzle design was used with a modified water phantom. The modification consisted of the addition of a small right circular cylinder inside the main water phantom and this small cylinder was used to score the proton absorbed dose as a function of depth along the central axis. This scoring cylinder had the same thickness as the outer one, a diameter of $20 \mathrm{~mm}$ and was sub-divided by planes placed $2 \mathrm{~mm}$ apart such that proton fluence could be scored as a function of depth. The absorbed dose was calculated using the cema approximation:

$$
D_{\mathrm{p}}=\int_{0}^{E_{\mathrm{p}}} \Phi_{\mathrm{p}}\left[\frac{1}{\rho} \frac{\mathrm{d} E_{\mathrm{p}}}{\mathrm{d} x}\right] \mathrm{d} E_{\mathrm{p}}
$$

where $D_{\mathrm{p}}$ is the proton absorbed dose (in Gy), $\Phi_{\mathrm{p}}$ is the distribution of proton fluence for a given energy and $\mathrm{d} E_{\mathrm{p}} / \rho \mathrm{d} x$ is the mass stopping power in water $\left(\mathrm{MeV} \mathrm{cm}^{2} \mathrm{~g}^{-1}\right)$ for protons of the respective energy. Stopping power values were taken from ICRU Publication 49 that were derived from the PSTAR database from Berger et al (2005).

2.1.5. Neutron contribution from nozzle components-Finally, the neutron contribution from each component along the proton beam central axis in the treatment nozzle was investigated using the same nozzle geometry shown in figure 1, but with a few modifications. Specifically, the detecting surfaces at different angles were eliminated and instead annular detecting volumes were placed at several locations on the beam central axis, including just downstream from the RMW, just downstream of the secondary scatterer and immediately upstream and downstream of the snout. The annuli were $1 \mathrm{~mm}$ thick right circular cylinders with radii ranging from 50 to $200 \mathrm{~mm}$. The annuli material was set to vacuum such that neither the proton beam nor the neutrons created were perturbed by them.

\section{Results and discussion}

\subsection{Neutron absorbed dose per therapeutic dose and neutron absorbed dose per source proton}

Figure 2(a) presents the neutron absorbed dose per therapeutic absorbed dose normalized to the scoring distance squared, which we will denote as $D_{\mathrm{n}} / D_{\mathrm{p}}$, and figure 2(b) shows the neutron absorbed dose per source proton, also normalized, or $D_{\mathrm{n}} / N_{\mathrm{p}}$, as a function of neutron emission angle. The angles represent the position of the detecting surfaces presented in figure 1. Note in both figures, 2(a) and (b), that $D_{\mathrm{n}} / D_{\mathrm{p}}$ and $D_{\mathrm{n}} / N_{\mathrm{p}}$ are larger at forward 
angles, decreasing rapidly with increasing angle. The larger dose at small angles is influenced by the presence of the water phantom, which also contributed to the neutron production. As the angle increased, the detecting surfaces were further away from the phantom and hence the phantom contribution diminished.

The value of $D_{\mathrm{n}} / D_{\mathrm{p}}$ is higher at forward angles smaller than $15^{\circ}$, for the largest treatment volume, followed by the $100 \times 100 \times 100 \mathrm{~mm}^{3}$ volume for which $D_{\mathrm{n}} / D_{\mathrm{p}}$ is slightly higher than for the smallest one, figure 2 . Between $15^{\circ}$ and $30^{\circ}$ there was a slight increase in $D_{\mathrm{n}} / D_{\mathrm{p}}$ for the smallest treatment volume which may have been due to the presence of the additional collimating material that was needed at the snout to produce smaller proton fields. For angles larger than $30^{\circ} D_{\mathrm{n}} / D_{\mathrm{p}}$ diminishes for all treatment volumes. The overall trend of higher dose with larger treatment volume is due in part to a larger contribution from the water phantom itself since relatively more protons reach the phantom and fewer stop in the final collimator.

An opposite behavior was observed for $D_{\mathrm{n}} / N_{\mathrm{p}}$ shown in figure 2(b) at forward angles. The higher $D_{\mathrm{n}} / N_{\mathrm{p}}$ was obtained for the smaller treated volume from angles between $5^{\circ}$ and $30^{\circ}$. In this case the last collimator was the predominating source of neutrons. The smaller the aperture, the more beam is intercepted in the collimator thus producing more secondary neutrons with higher energies. However, for smaller fields the contribution from the RMW and the water phantom is less because the proton beam interacts with less RMW material and at the same time that fewer protons are needed to obtain the desired SOBP width.

\subsection{Neutron energy spectra}

As shown in figure 3(a) to (c), the neutron production for angles less than $30^{\circ}$ was uniform from a few $\mathrm{MeV}$ to around $160 \mathrm{MeV}$. Above these energies, the neutron fluence per solid angle per $\mathrm{MeV}$ decreased. At larger emission angles, the neutron production was less uniform and decreased sharply with neutron energy. There were a significant number of neutrons created at energies below a few $\mathrm{MeV}$ for all angles and we attributed this to the near isotropic production of evaporation neutrons. Even though these neutrons have low energy and generate little absorbed dose per neutron, they should be included in dosimetric considerations as their fluence is high. For instance, Yan et al (2002) observed a significant dose equivalent contribution from such evaporation neutrons for a $160 \mathrm{MeV}$ proton beam. Similar neutron spectra have been predicted at other proton energies (Yan et al 2002, Fontenot et al 2005, Newhauser et al 2002).

\subsection{Neutron contribution from nozzle components}

Figure 4 shows the neutron fluence per source proton at various locations downstream of the nozzle as a function of the radial distance from the central axis for each treatment field considered. Figures 4(a) and (b) present the neutron fluence per source particle just downstream from the RMW and the secondary scatterer, respectively. Both figures show a higher neutron production for the $150 \times 150 \times 150 \mathrm{~mm}^{3}$ treatment volume configuration. This result was expected since the largest areal densities of PMMA and lead were used in the nozzle for this treatment volume. 
Figures 4(c) and (d) show the neutron fluence per source particle immediately upstream and downstream of the snout. The neutron fluence per source particle is around a factor of 3-20 smaller downstream from the snout than upstream, and the largest difference was found for the smallest treatment volume. This increase in neutron fluence before the snout is attributed to backscattering. Several simulations were run to test this backscatter effect. In the simulations the density of copper was varied and the neutron fluence upstream of the snout was found to decrease considerably for the lower densities used.

In summary, the largest neutron fluence was found after the RMW for all radii studied with the exception of the backscatter effect. The neutron fluence downstream of the RMW decreases with distance from the RMW and for smaller treatment volumes the neutron fluence downstream of the RMW is not significantly higher than those detected downstream of the snout. This shows the competitive effect between both sources when small treatment volumes are irradiated.

Meanwhile, the interactions with the RMW produced the largest fluence of high-energy neutrons. Figure 5 presents the neutron energy spectra for the largest treatment volume at 25 $\mathrm{mm}$ radial distance from the central axis for the four positions studied. A significant number of high-energy neutrons were found up to a radial distance of $100 \mathrm{~mm}$, indicating a predominant neutron production in the forward direction. The relative contribution to fluence of these high-energy neutrons decreases along the central axis and with increasing radial distance.

The neutron contribution to fluence by the final snout was also studied for the smaller treatment volume and it is presented in figure 6. During the simulations the material of the final aperture was changed to vacuum. This allowed the determination of the influence of the final aperture over the final neutron fluence at the snout exit. The neutron fluence just downstream of the snout increases by approximately a factor of 5 due to the snout's presence.

Also, it is relevant to mention that although the neutron contribution from other collimating components was not individually studied, their contribution was accounted for in the neutron absorbed dose calculations previously reported. The neutron contribution from the snout was studied in more detail because this is the component of the collimating system that interacts the most with the proton beam.

Overall, the RMW is the main source of neutrons within the nozzle, contributing also the largest fluence of energetic neutrons. Therefore, it may be important to characterize the neutron production in the RMW in order to refine shielding designs for the nozzle and the treatment vault. On the other hand, due to the neutron fluence dependence on distance from the source, neutrons created in the RMW will not contribute significantly to the dose at isocenter. Most of the neutron dose to the patient is due to interactions of the spread beam with the final collimating aperture. 


\subsection{Uncertainty}

In general, a statistical uncertainty of approximately $5 \%$ was obtained in the results for the energy range of interest. This uncertainty is related to the statistical fluctuations in the Monte Carlo simulations and not with systematic uncertainties.

\section{Conclusion}

From the simulations performed we determined that the RMW is the nozzle's component that produces the most neutrons because it possesses the largest areal density of high- $Z$ and low- $Z$ material intercepting the proton beam. Such neutrons are forward directed with energies as high as the primary proton beam energy. Their production decreases with emission angle and distance from the main source of neutrons, RMW, increases. This suggests that the design of the appropriate room and nozzle shielding will be determined primarily by the RMW. At the patient's location the predominant source of neutrons comes from the snout. The RMW will also contribute neutrons but due to its distance from the patient this is not as significant. Although the snout contributes to backscattered neutrons, this is not directly relevant to the secondary dose to the patient. This should however be considered for the shielding of the treatment unit. An isotropic emission of evaporation neutrons was also observed. The proportion of absorbed dose from evaporation neutrons was small, but the neutron's fluence intensity was non-negligible for all angles studied. The presence of the water phantom contributed to the production of neutrons as well.

The data presented here provide insight to the determination of the neutron production at a given therapeutic dose and a given distance from the nozzle for similar nozzle configurations at $200 \mathrm{MeV}$ proton energy. Varying components or conditions, for instance the secondary scatterer or the beam energy, will yield different neutron production ergo different absorbed dose. Nevertheless this work presents how each nozzle component contributes to the neutron production. Knowing the location and intensity of neutron sources inside the radiation treatment head may help guide the design of better local shielding. Tayama et al (2006) reported that by adding a pre-collimator upstream in the nozzle head it was possible to diminish the neutron equivalent dose per therapeutic dose. A similar study by Taddei et al (2008) showed that the addition of several shielding materials placed at different positions within the nozzle can reduce the stray radiation that reaches the patient. However, they did not attempt to localize the neutron sources and therefore could not design a low-mass shield that was suitable for installation on a rotating gantry. Based on the current results, it appears that designing a local neutron shield around the RMW is the next logical step toward achieving clinically practical improvements in the shielding of the treatment head.

\section{Acknowledgments}

We thank the National Science Foundation for funding the University of Wisconsin-managed Grid Laboratory of Wisconsin (GLOW) computer cluster (NSF award number 0320708) that was used for these simulations. This work was funded in part by a National Institute of Health Ruth L Kirschstein National Research Service Award Individual Predoctoral Fellowship Program (award number F31 CA119943-03) to A Pérez-Andújar and P M DeLuca Jr and by Northern Illinois University through a Department of Defense subcontract (contract W81XWH-08-1-0205) to W D Newhauser. 


\section{References}

Agosteo S, Birattari C, Caravaggio M, Silari M, Tosi G. Secondary neutron and photon dose in proton therapy. Radiother Oncol. 1998; 48:293-305. [PubMed: 9925249]

Berger, MJ.; Coursey, JS.; Zucker, MA.; Chang, J. ESTAR, PSTAR, and ASTAR: computer programs for calculating stopping-power and range tables for electrons, protons, and helium ions. National Institute of Standards and Technology; Gaithersburg, MD: 2005. version 1.2.3

Bertini HW. Low-energy intranuclear cascade calculation. Phys Rev. 1963; 131:1801-21.

Brada M, Pijls-Johannesma M, De Ruysscher D. Proton therapy in clinical practice: current clinical evidence. J Clin Oncol. 2007; 25:965-70. [PubMed: 17350945]

Chadwick MB, et al. Cross-section evaluations to $150 \mathrm{MeV}$ for accelerator-driven systems implementation in MCNPX. Nucl Sci Eng. 1999; 131:293-328.

Chu WT, Ludewigt BA, Renner TR. Instrumentation for treatment of cancer using proton and light-ion beams. Rev Sci Instrum. 1993; 64:2055-2122.

Fontenot J, Taddei P, Zheng Y, Mirkovic D, Jordan T, Newhauser W. Equivalent dose and effective dose from stray radiation during passively scattered proton radiotherapy for prostate cancer. Phys Med Biol. 2008; 53:1-12. [PubMed: 18182684]

Fontenot JD, Newhauser WD, Titt U. Design tools for the proton therapy nozzles based on the double scattering foil technique. Radiat Prot Dosim. 2005; 116:211-15.

Gottschalk B, Koehlera AM, Schneider RJ, Sisterson JM, Wagner MS. Multiple Coulomb scattering of $160 \mathrm{MeV}$ protons. Nucl Instrum Methods B. 1993; 74:467-90.

Gottschalk, B.; Koehler, A.; Sisterson, J.; Wagner, M. The case for passive beam spreading. Proc Radiotherapy Workshop; Switzerland. 29 February-1 March; Paul Scherrer Institute; 1991. p. 50Report No. 111

Gragoudas ES, Lane AM, Munzenrider J, Egan KM, Li W. Long-term risk of local failure after proton therapy for choroidal/ciliary body melanoma. Trans Am Ophthalmol Soc. 2002; 100:43-9. [PubMed: 12545676]

Grid Laboratory of Wisconsin (GLOW). Department of medical physics university of WisconsinMadison. 2004. http://www.medphysics.wisc.edu/glow/history.html

Hérault J, Iborra N, Serrano B, Chauvel P. Monte Carlo simulations of a proton therapy platform devoted to ocular melanoma. Med Phys. 2005; 32:910-19. [PubMed: 15895573]

International Commission on Radiation Units and Measurements (ICRU). ICRU. Bethesda, MD: 1993. Stopping Powers and Ranges for Protons and Alpha Particles. Report 49

International Commission on Radiation Units and Measurements (ICRU). ICRU. Bethesda, MD: 1998. Proton Dosimetry: Part 1. Beam Production, Beam Delivery and Measurement of Absorbed Dose. Report 59

International Commission on Radiation Units and Measurements (ICRU). ICRU. Bethesda, MD: 2000. Nuclear Data for Neutron and Proton Radiotherapy and for Radiation Protection. Report 63

International Commission on Radiation Units and Measurements (ICRU). ICRU. Bethesda, MD: 2007. Prescribing, Recording, Reporting Proton-Beam Therapy. Report 78

Janni JF. Proton range-energy tables, 1 keV-10 GeV. At Data Nucl Data Tables. 1982; 27:147.

Koch N, Newhauser WD. Virtual commissioning of a treatment planning system for proton therapy of ocular cancers. Radiat Prot Dosim. 2005; 115:159-63.

Koch N, Newhauser W, Titt U, Gombos D, Coombes K, Starkschall G. Monte Carlo calculations and measurements of absorbed dose per monitor unit for the treatment of uveal melanoma with proton therapy. Phys Med Biol. 2008; 53:1581-94. [PubMed: 18367789]

Koehler AM, Schneider RJ, Sisterson JM. Range modulation for protons and heavy ions. Nucl Instrum Methods. 1975; 131:437-40.

Lodge M, Pijls-Johannesma M, Stirk L, Munro AJ, De Ruysscher D, Jefferson T. A systematic literature review of the clinical and cost-effectiveness of hadron therapy in cancer. Radiother Oncol. 2007; 83:110-22. [PubMed: 17502116]

Matsuda, K.; Umezawa, M.; Fujimaki, H.; Nishiuchi, H.; Yanagisawa, M.; Tadokoro, M.; Sakae, T.; Terunuma, T.; Akine, Y. Proton Medical Research Center Progress Report 2000-2005 Takaji 
Sake. Tsukuba: University of Tsukuba; 2006. Development of irradiation system with a range modulation wheel and a contoured second scatterer; p. 213-20.

Miller DW. A review of proton beam radiation therapy. Med Phys. 1995; 22:1943-54. [PubMed: 8587548]

Newhauser W, Fontenot J, Zheng Y, Polf J, Titt U, Koch N, Zhang X, Mohan R. Monte Carlo simulations for configuring and testing and analytical proton dose-calculation algorithm. Phys Med Biol. 2007; 52:4569-84. [PubMed: 17634651]

Newhauser W, Koch N, Hummel S, Ziegler M, Titt U. Monte Carlo simulations of a nozzle for the treatment of ocular tumours with high-energy proton beams. Phys Med Biol. 2005; 50:5229-49. [PubMed: 16264250]

Newhauser WD, Titt U, Dexheimer D, Yan X, Nill S. Neutron Shielding verification measurements and simulations for a $235 \mathrm{MeV}$ proton therapy center. Nucl Instrum Methods A. 2002; 476:80-4.

Paganetti H. Nuclear interactions in proton therapy: dose and relative biological effect distributions originating from primary and secondary particles. Phys Med Biol. 2002; 47:747-64. [PubMed: 11931469]

Pelowiz, DBE. MCNPX User's Manual. Los Alamos, NM: Los Alamos National Laboratory; 2005. Version 2.5.0 LA-CP-05-0369

Polf JC, Harvey MC, Titt U, Newhauser WD, Smith A. Initial beam size study for passive scatter proton therapy I. Monte Carlo verification. Med Phys. 2007; 34:4213-18. [PubMed: 18072485]

Polf JC, Newhauser WD. Calculations of neutron dose equivalent exposures from range-modulated proton therapy beams. Phys Med Biol. 2005; 50:3859-73. [PubMed: 16077232]

Schneider U, Agosteo S, Pedroni E. Secondary neutron dose during proton therapy using spot scanning. Int J Radiat Oncol Biol Phys. 2002; 53:244-51. [PubMed: 12007965]

Suit HD. Local control and patient survival. Int J Radiat Oncol Biol Phys. 1992; 23:653-60. [PubMed: 1612967]

Taddei PJ, Fontenot JD, Zheng Y, Mirkovic D, Lee AK, Titt U, Newhauser WD. Reducing stray radiation dose to patients receiving passively scattered proton radiotherapy for prostate cancer. Phys Med Biol. 2008; 53:2131-47. [PubMed: 18369278]

Tayama R, Fujita Y, Tadokoro M, Fujimaki H, Sakae T, Terunuma T. Measurement of neutron dose distribution for a passive scattering nozzle at the Proton Medical Research Center (PMRC). Nucl Instrum Methods A. 2006; 564:532-36.

Titt U, Newhauser WD. Neutron shielding calculations in a proton therapy facility based on Monte Carlo simulations and analytical models: criterion for selecting the method of choice. Radiat Prot Dosim. 2005; 115:144-8.

Titt U, Zheng Y, Vassiliev ON, Newhauser WD. Monte Carlo investigations of collimator scatter of proton-therapy beams produced using the passive scattering method. Phys Med Biol. 2008; 53:487-504. [PubMed: 18185001]

Wilson RR. Radiological use of fast protons. Radiology. 1946; 47:487-91. [PubMed: 20274616]

Yan X, Titt U, Koehler AM, Newhauser WD. Measurement of neutron dose equivalent to proton therapy patients outside of the proton radiation field. Nucl Instrum Methods A. 2002; 476:429-34.

Zheng Y, Fontenot J, Taddei P, Mirkovic D, Newhauser W. Monte Carlo simulations of neutron spectral fluence, radiation weighting factor and ambient dose equivalent for a passive scattered proton therapy unit. Phys Med Biol. 2008; 53:187-201. [PubMed: 18182696]

Zheng Y, Newhauser W, Fontenot J, Koch N, Mohan R. Monte Carlo simulations of stray neutron radiation exposures in proton therapy. J Nucl Mat. 2007a; 361:289-97.

Zheng Y, Newhauser WD, Fontenot JD, Taddei PJ, Mohand R. Monte Carlo study of neutron dose equivalent during passive scattering proton therapy. Phys Med Biol. 2007b; 52:4481-96.

[PubMed: 17634645] 

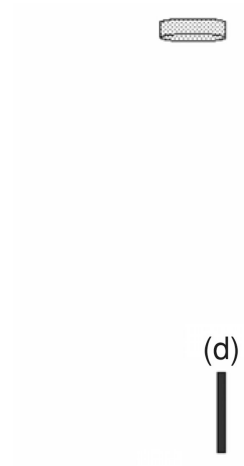

(a)

(b)

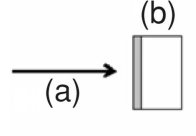

d) (e) (f)

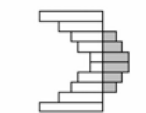

(c)

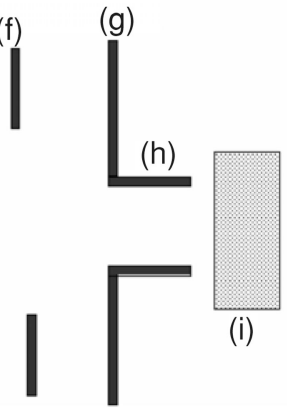

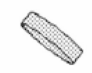
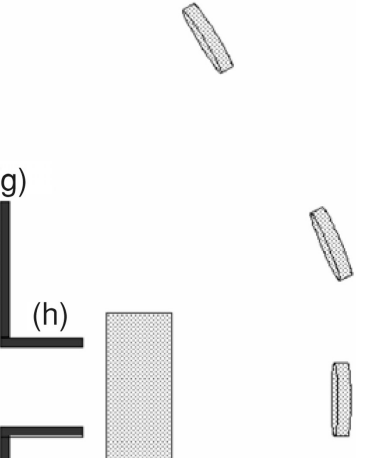

Figure 1.

Schematic diagram of the proton treatment nozzle simulated with MCNPX. The arrow (a) shows the direction of the monoenergetic $200 \mathrm{MeV}$ proton beam. Also shown are (b) the RMW, (c) the second scatterer, the (d) first, (e) second and (f) third collimator set, (g) the snout's collimator, (h) the snout aperture, (i) the stopping-length water phantom (the phantom is long enough such that the proton beam stops in it) and (j) the neutron detecting surfaces. 

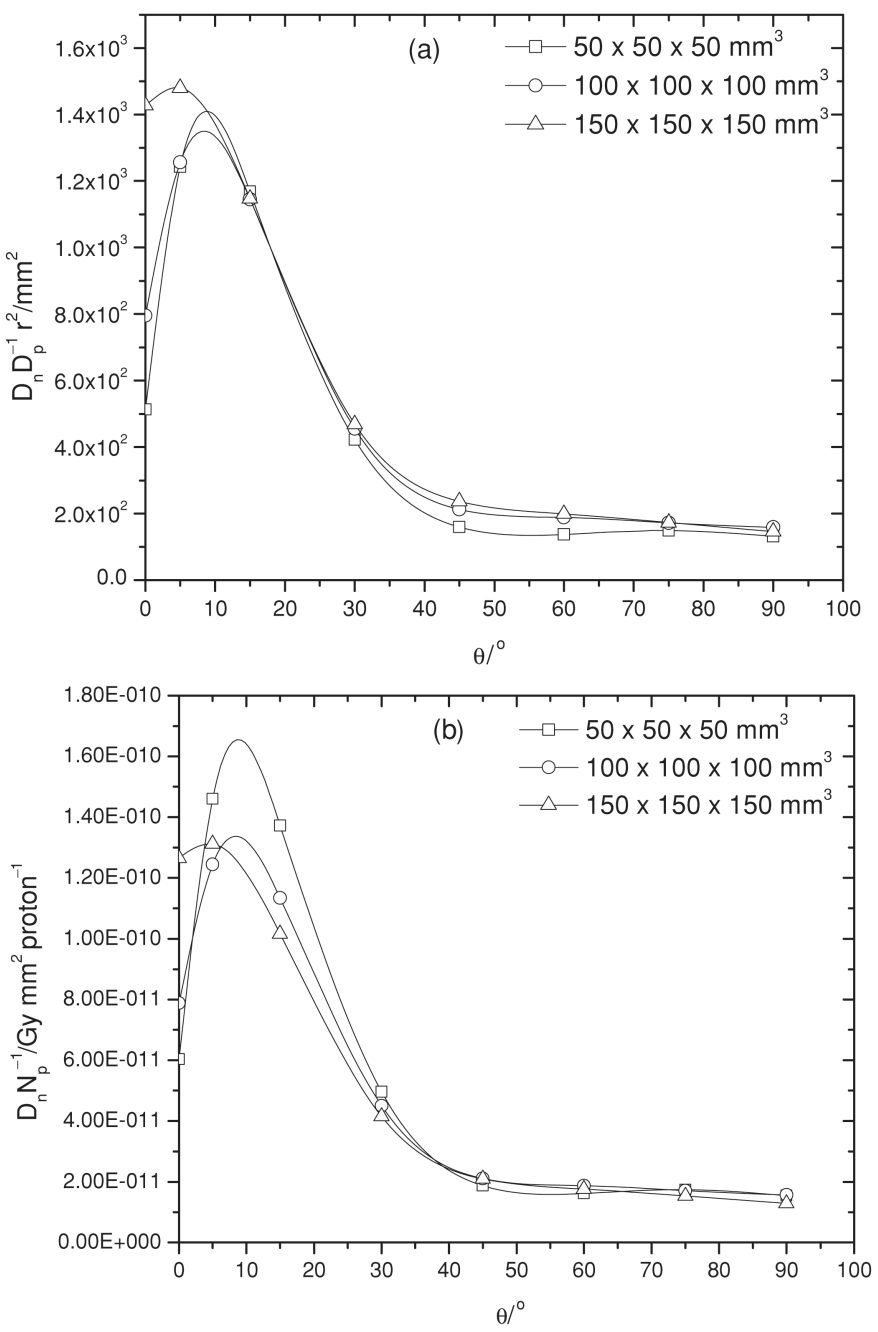

Figure 2.

(a) Neutron absorbed dose per therapeutic absorbed dose multiplied by the distance square $\left(D_{\mathrm{n}} r^{2} / D_{\mathrm{p}}\right)$ and (b) neutron absorbed dose per source proton $\left(D_{\mathrm{n}} r^{2} / N_{\mathrm{p}}\right)$ for the three treatment volumes studied as a function of the neutron emission angle, $\theta$, normalized by the distance square from the RMW interface to the detecting surface, $4.06 \mathrm{~m}$. 

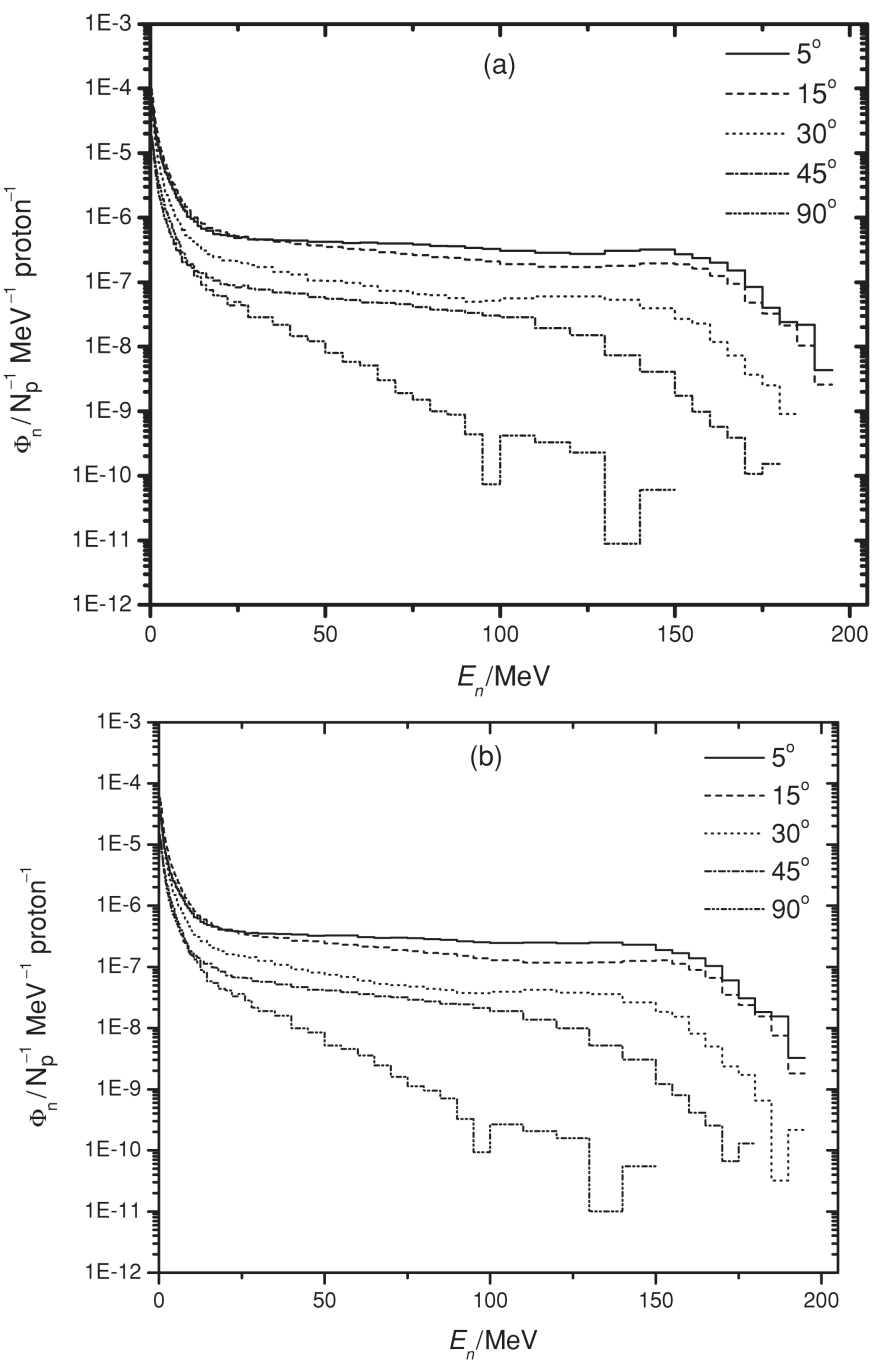


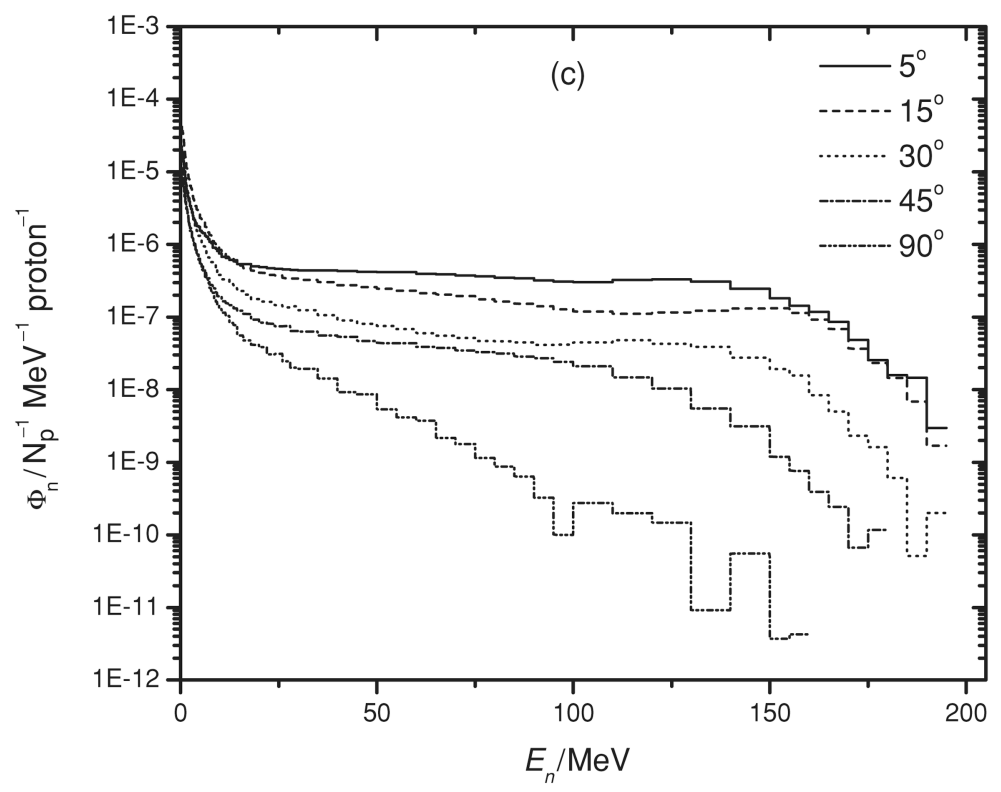

Figure 3.

Neutron energy spectra, $\Phi_{\mathrm{n}}$, as a function of neutron energy, $E_{\mathrm{n}}$, for the three studied volumes, (a) $50 \times 50 \times 50 \mathrm{~mm}^{3}$, (b) $100 \times 100 \times 100 \mathrm{~mm}^{3}$ and (c) $150 \times 150 \times 150 \mathrm{~mm}^{3}$ at various emission angles. 

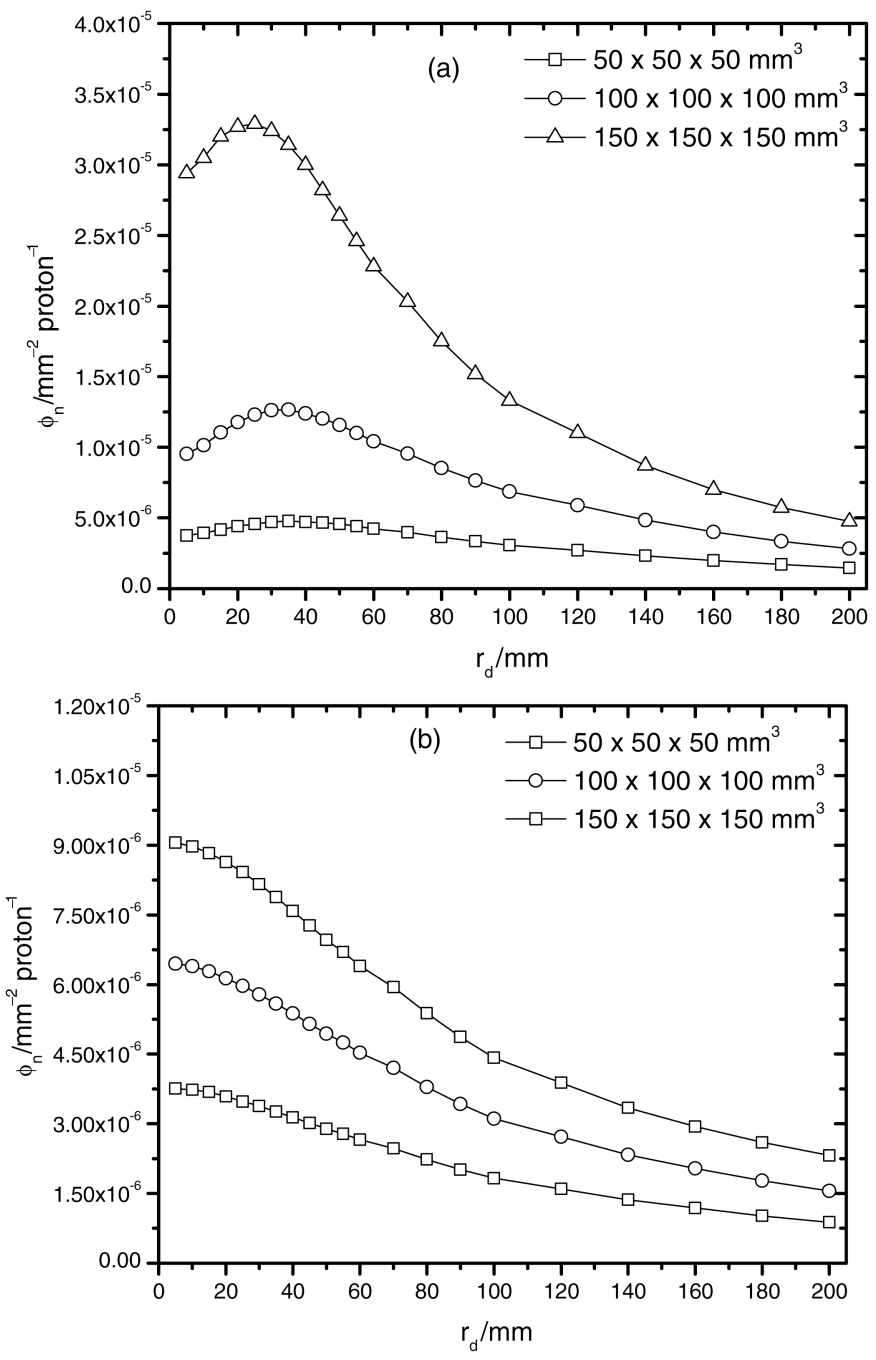

Phys Med Biol. Author manuscript; available in PMC 2014 August 18. 

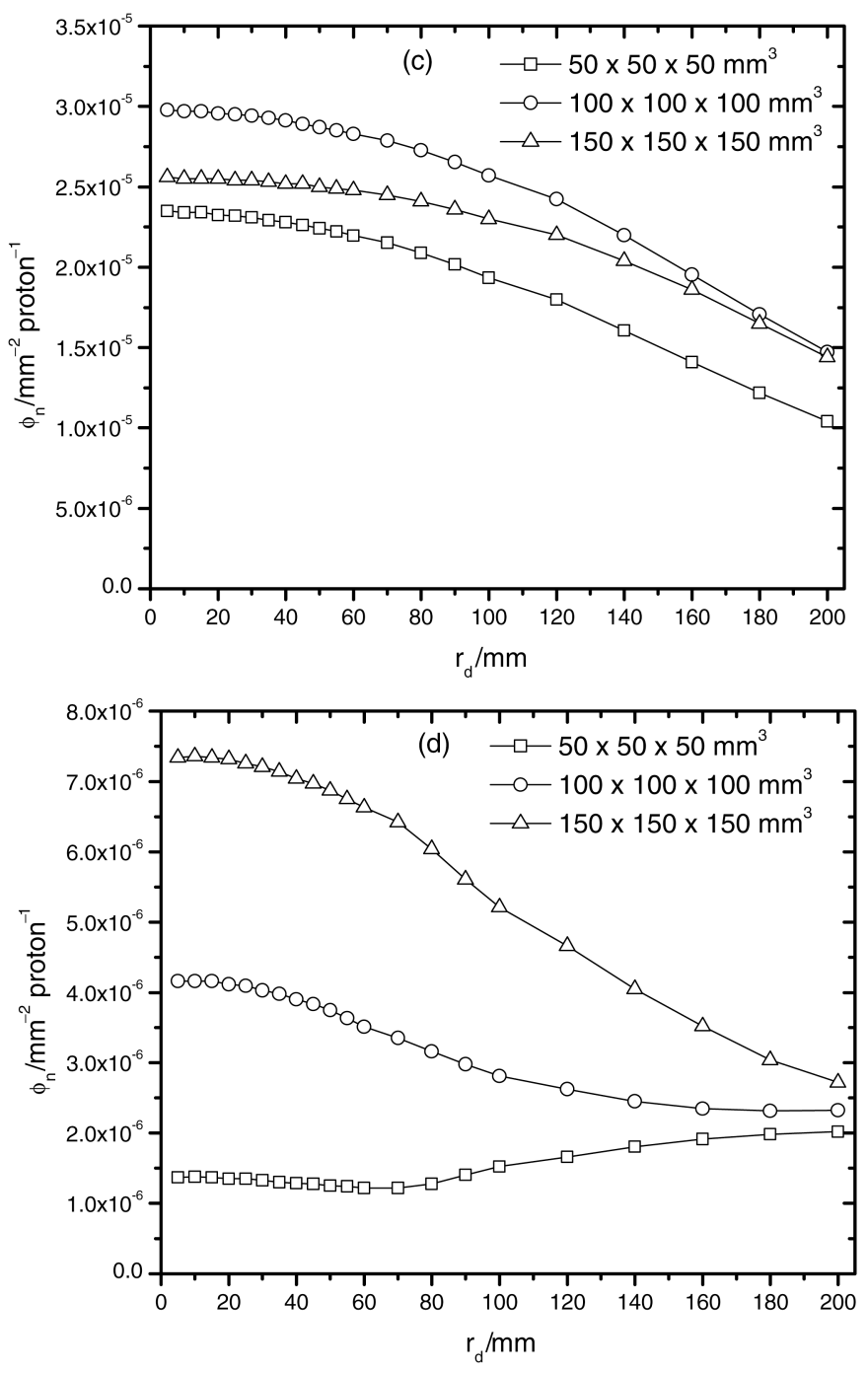

Figure 4.

Total neutron fluence, $\phi_{\mathrm{n}}$, as a function of radial distance $r_{\mathrm{d}}$ for the three studied volumes (a) just downstream from the RMW and (b) the second scatterer, (c) upstream and (d) downstream (d) of the snout. 


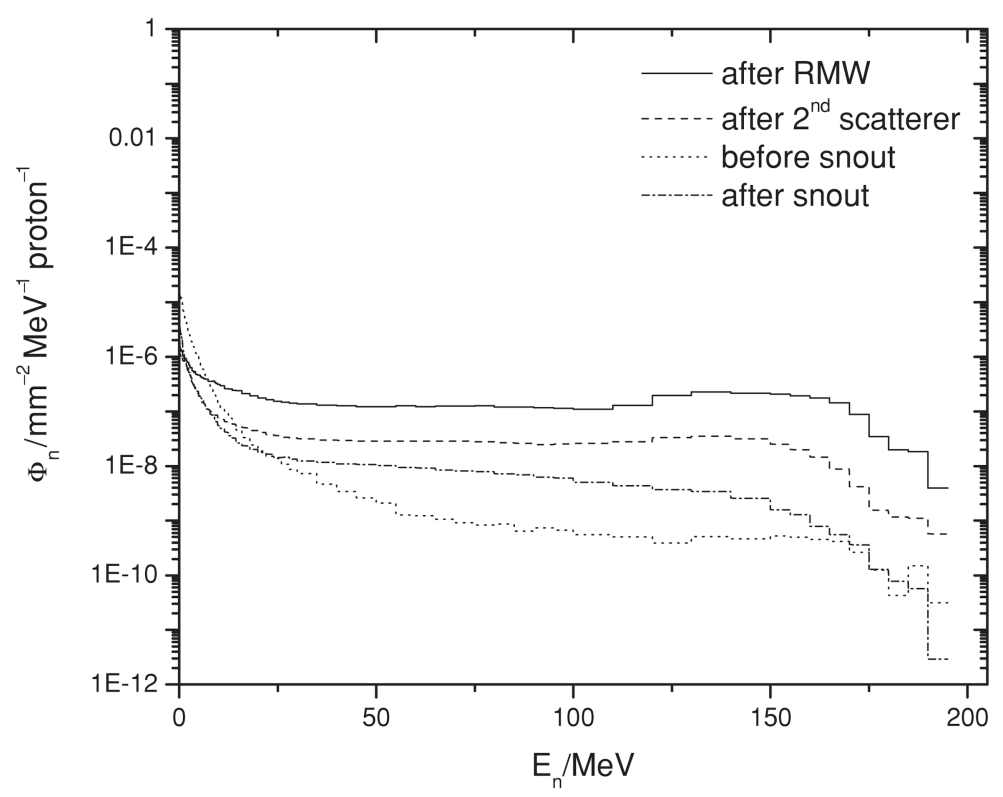

Figure 5.

Neutron energy spectra, $\Phi_{\mathrm{n}}$, as a function of neutron energy, $E_{\mathrm{n}}$, at a radial distance of 25 $\mathrm{mm}$ from the central axes at the four positions studied for the $150 \times 150 \times 150 \mathrm{~mm}^{3}$ treatment volume: just downstream from the RMW (after RMW) and second scatterer (after second scatterer), just upstream and downstream of the snout (before snout and after snout, respectively). 


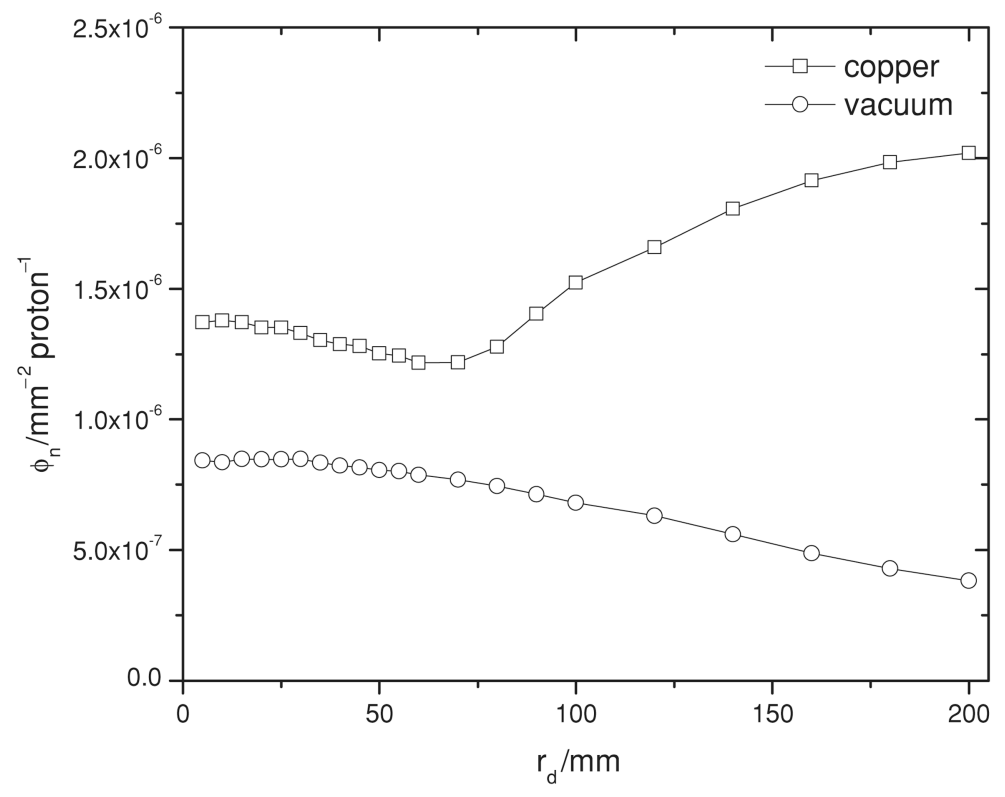

Figure 6.

Neutron fluence, $\varphi$ n as a function of radial distance, $r$ d, downstream of the snout when the material of the snout is set to copper and when set to vacuum. This was done to test the neutron contribution from the snout to the neutron fluence just downstream of it. 


\section{Table 1}

Nozzle components and their respective distances from the RMW interface and thicknesses. The collimating components had an extension of $100 \mathrm{~mm}$ from the internal field definition with the exception of the snout apperture that has a fixed extension of $60 \mathrm{~mm}$. The characteristics of the secondary scatterer are given in table 3.

\begin{tabular}{llr}
\hline Component & Distance $(\mathbf{m})$ & Thickness $(\mathbf{m m})$ \\
\hline Secondary scatterer junction & 0.856 & - \\
First set of collimators & 1.045 & 70 \\
Second set of collimators & 1.558 & 120 \\
Third set of collimators & 2.064 & 60 \\
Snout's collimator & 2.550 & 60 \\
Final snout aperture & 2.610 & 250 \\
\hline
\end{tabular}




\section{Table 2}

Areal density for the steps used to degrade the energy of the beam and obtained the desire SOBP for each of the treatment volumes simulated. The first eight steps were used for a $50 \mathrm{~mm}$ SOBP, the first 14 for the 100 $\mathrm{mm}$ and finally the 21 steps were used for the $150 \mathrm{~mm}$ SOBP.

\begin{tabular}{rrr}
\hline Step number & PMMA $\left(\mathbf{k g ~ m}^{-\mathbf{2}}\right)$ & Lead $\left(\mathbf{k g ~ m}^{-2}\right)$ \\
\hline 1 & 2.9 & 32.8 \\
2 & 13.0 & 28.5 \\
3 & 21.5 & 27.1 \\
4 & 29.8 & 26.1 \\
5 & 38.6 & 24.2 \\
6 & 47.1 & 22.7 \\
7 & 55.8 & 21.1 \\
8 & 64.2 & 19.8 \\
9 & 72.7 & 18.4 \\
10 & 81.6 & 16.4 \\
11 & 90.4 & 14.5 \\
12 & 99.1 & 12.8 \\
13 & 107.8 & 11.0 \\
14 & 116.8 & 8.6 \\
15 & 125.6 & 6.8 \\
16 & 134.5 & 4.6 \\
17 & 143.8 & 1.9 \\
18 & 152.5 & - \\
19 & 160.3 & - \\
20 & 168.0 & - \\
21 & 175.8 &
\end{tabular}

Phys Med Biol. Author manuscript; available in PMC 2014 August 18. 


\section{Table 3}

Second scatterer areal thickness as a function of the radial distance from the central axis. Each layer of the high- $Z$ portion of the second scatterer was modeled as a disk and each layer of the low- $Z$ was modeled as an annulus.

\begin{tabular}{rrr}
\hline Radius $(\mathbf{m m})$ & PMMA $\left(\mathbf{k g ~ m}^{-\mathbf{2}}\right)$ & Lead $\left(\mathbf{k g ~ m}^{-\mathbf{2}}\right)$ \\
\hline 0.1 & 0.00 & 29.40 \\
5.04 & 0.91 & 27.72 \\
10.08 & 3.37 & 23.18 \\
15.12 & 6.81 & 17.32 \\
20.16 & 10.47 & 10.05 \\
25.20 & 13.37 & 4.65 \\
30.24 & 15.11 & 1.44 \\
35.28 & 15.89 & 0.00 \\
40.31 & 15.89 & 0.00 \\
45.35 & 15.89 & 0.00 \\
\hline
\end{tabular}

